\title{
Rationale and design of the EPCHF trial: the early palliative care in heart failure trial (EPCHF)
}

\author{
Marc Ulrich Becher ${ }^{1}$ (1) $\cdot$ Mahmoud Balata $^{1} \cdot$ Michaela Hesse $^{2} \cdot$ Fabian Draht $^{3} \cdot$ Christian Zachoval $^{1}$. \\ Birgitta Weltermann ${ }^{3} \cdot$ Ralf Westenfeld ${ }^{4} \cdot$ Martin Neukirchen $^{5} \cdot$ Roman Pfister $^{6} \cdot$ Thomas Standl $^{7} \cdot$ Lukas Radbruch $^{2}$. \\ Georg Nickenig'
}

Received: 5 April 2021 / Accepted: 23 June 2021 / Published online: 9 July 2021

(c) The Author(s) 2021

\begin{abstract}
The progressive nature of heart failure (HF) coupled with high mortality and poor quality-of-life (QoL) mandates greater attention to palliative care (PC) as a routine component of HF management. Limited evidence exists from randomized controlled trials supporting the use of interdisciplinary palliative care in the progressive course of HF. The early palliative care in heart failure trial (EPCHF) is a prospective, controlled, nonblinded, multicenter study of an interdisciplinary palliative care intervention in 200 patients with symptomatic HF characterized by NYHA $\geq 2$. The 12-month EPCHF intervention includes monthly consultations by a palliative care team focusing on physical and psychosocial symptom relief, attention to spiritual concerns and advance care planning. The primary endpoint is evaluated by health-related QoL questionnaires after 12 months of treatment. First the functional assessment of chronic illness therapy palliative care (FACIT-Pal) score evaluating QoL living with a chronic disease and second the Kansas City cardiomyopathy questionnaire (KCCQ) measuring QoL living with heart failure will be determined. Secondary endpoints are changes in anxiety/depression (HADS), symptom burden score (MIDOS), spiritual well-being functional assessment of chronic illness therapy spiritual well-being scale (FACIT-Sp), medical resource and cost assessment. EPCHF will help evaluate the efficacy and cost-effectiveness of palliative care in symptomatic HF using a patient-centered outcome as well as clinical and economic endpoints. EPCHF is funded by the Bundesministerium für Bildung und Forschung (BMBF, 01GY17).
\end{abstract}

Keywords Palliative care $\cdot$ Heart failure $\cdot$ Quality of life $\cdot$ FACIT-PAL $\cdot$ KCCQ

Marc Ulrich Becher

ubecher@uni-bonn.de

1 Department of Cardiology, Angiology, Pneumology and Medical Intensive Care, University Hospital Bonn, Venusberg-Campus 1, 53127 Bonn, Germany

2 Department of Palliative Medicine, University Hospital Bonn, Bonn, Germany

3 Institute of General Practice and Family Medicine, University Hospital Bonn, Bonn, Germany

4 Department of Cardiology, University Hospital Düsseldorf, Düsseldorf, Germany

5 Interdisciplinary Centre for Palliative Care, University Hospital Düsseldorf, Düsseldorf, Germany

6 Department of Cardiology, University Hospital Cologne, Cologne, Germany

7 Department of Anesthesia, Intensive Care and Palliative Medicine, Städtisches Klinikum Solingen, Solingen, Germany

\begin{tabular}{|c|c|}
\hline \multicolumn{2}{|c|}{ Abbreviations } \\
\hline BMBF & Bundesministerium für Bildung und \\
\hline & Forschung \\
\hline BNP & B-type natriuretic peptide \\
\hline EPCHF & Early palliative care in heart failure trial \\
\hline FACIT-Pal & $\begin{array}{l}\text { Functional assessment of chronic illness } \\
\text { therapy palliative care }\end{array}$ \\
\hline FACIT-Sp & $\begin{array}{l}\text { Functional assessment of chronic illness } \\
\text { therapy spiritual well-being scale }\end{array}$ \\
\hline $\mathrm{HF}$ & Heart failure \\
\hline KCCQ & Kansas City cardiomyopathy questionnaire \\
\hline HADS & Hospital anxiety and depression scale \\
\hline LVEF & Left ventricular ejection fraction \\
\hline MIDOS & Minimal documentation system \\
\hline NT-proBNP & $\mathrm{N}$-terminal pro-B-type natriuretic peptide \\
\hline NYHA & New York Heart Association \\
\hline $\mathrm{PC}$ & Palliative care \\
\hline QoL & Quality of life \\
\hline
\end{tabular}




\section{Background and rationale}

The epidemiological burden of heart failure (HF) is tremendous, with an estimated 26 million people affected worldwide [1]. HF currently affects over 2 million patients in Germany. Accordingly, HF was the most common cause of disease-related hospitalization, the second leading cause of hospital stay, and the leading cause of in-hospital deaths in Germany in 2013 [1]. Despite recent therapeutic advances, HF is a debilitating syndrome that results in a high burden of symptoms and poor QoL. Patients suffer not only from physical effects but also from psychosocial and spiritual distress [2, 3]. Selected HF patients are candidates for aggressive treatments, such as cardiac transplantation or mechanical circulatory support; nevertheless, the application of these therapies to the broader HF population is limited by constrained resources [4]. The progressive nature of HF coupled with high mortality rates and poor QoL mandates greater attention to palliative care (PC) as a routine component of HF management [5].

PC with its focus on management of symptoms, psychosocial support, and assistance with decision making has the potential to improve the quality of care and reduce the use of medical services [3, 4]. However, PC has traditionally been delivered late in the course of disease to patients who are hospitalized in specialized inpatient units or as a consultative service for patients with uncontrolled symptoms [5, 6]. Previous studies have suggested that late referrals to palliative care are inadequate to alter the quality and delivery of care provided to patients $[7,8]$. To have a meaningful effect on patients' QoL and end-of-life care, PC services should be provided earlier in the course of the disease. We here postulate that introducing of PC earlier is feasible and beneficial among patients with symptomatic $\mathrm{HF}$ in the outpatient setting.

PC is a multidisciplinary approach that focuses on providing patients with relief from symptoms, pain, and stress of living with a serious illness at any stage of disease [6]. The goal of the current study is to examine the effect of early integration of PC within standard cardiac care on QoL, reported cardiac outcomes, and the use of health services among HF patients.

We hypothesized that patients who received early PC in the ambulatory care setting, as compared with patients who received standard cardiac care alone, would have a better QoL due to lower rates of depressive symptoms, symptom burden, unmet spiritual needs, reported cardiac outcomes and less aggressive end-of-life care thereby improving QoL for both, patients and their caregivers.

Despite consensus-panel and guideline recommendations the integration of $\mathrm{PC}$ with evidence-based HF therapies in the later stages of disease is limited [9, 10]. Many physicians equate $\mathrm{PC}$ with end-of-life care and prognostic uncertainty poses a challenge in choosing the appropriate time point to implement PC. Furthermore, cardiologists typically lack formalized training in the PC-principles and it is less clear which HF patients benefit from palliative interventions and which interventions improve QoL achieving outcomes desired by patients and family members [9]. However, multiple recent pilot studies in HF populations have suggested that PC may reduce symptom burden and improve QoL [11-13]. These studies have highlighted the importance of symptoms, such as anxiety and depression in HF patients in addition to the commonly recognized symptoms of fatigue, dyspnea and nausea. Moreover, a randomized trial of PC in Stage 4 lung cancer showed improved patient's symptoms, improves QoL and patients randomized to PC survived longer than those randomized to usual care [14]. Furthermore, multiple studies in oncology and noncancer illness patients have shown reduced costs leading to increased interest of whether similar results can be found in other common diseases, such as HF [15-17]. These studies provide the foundation and rationale for a large-scale randomized HF trial sufficiently powered to assess the effect of early palliative care on clinical outcomes in HF patients.

To help create this body of evidence, the BMBF has funded the early palliative care in heart failure trial (EPCHF) to evaluate if a multidimensional palliative care intervention improves health-related outcomes relative to usual care alone in symptomatic HF patients (Registration No.: DRKS Register 00013922, Studiencode: MED2-201604_EPCHF, Sponsor: BMBF FKZ: 01GY1704). This article describes the design and rationale of the EPCHF trial (https://www. gesundheitsforschung-bmbf.de/de/epchf-studie-fruhe-palli ativmedizinische-intervention-bei-patientinnen-und-patie nten-mit-7630.php). The authors are solely responsible for the design and conduct of this study, the drafting and editing of this paper and its final contents. Of note, the BMBF is currently funding multiple clinical trials and research projects related to palliative care (see https://www.bmbf.de).

\section{Methods}

\section{Trial design}

EPCHF is a prospective, controlled, two-arm, non-blinded, randomized, multicenter clinical trial of early palliative care integrated with standard cardiac care, as compared with standard cardiac care alone (Fig. 1). The multicenter study will be performed at the departments of Internal Medicine and Palliative Care of the University Hospital of Bonn and departments of Internal Medicine and Palliative Care of the University Hospital of Düsseldorf. Eligible patients with symptomatic HF and dyspnea NYHA $\geq 2$ will be randomly assigned to 


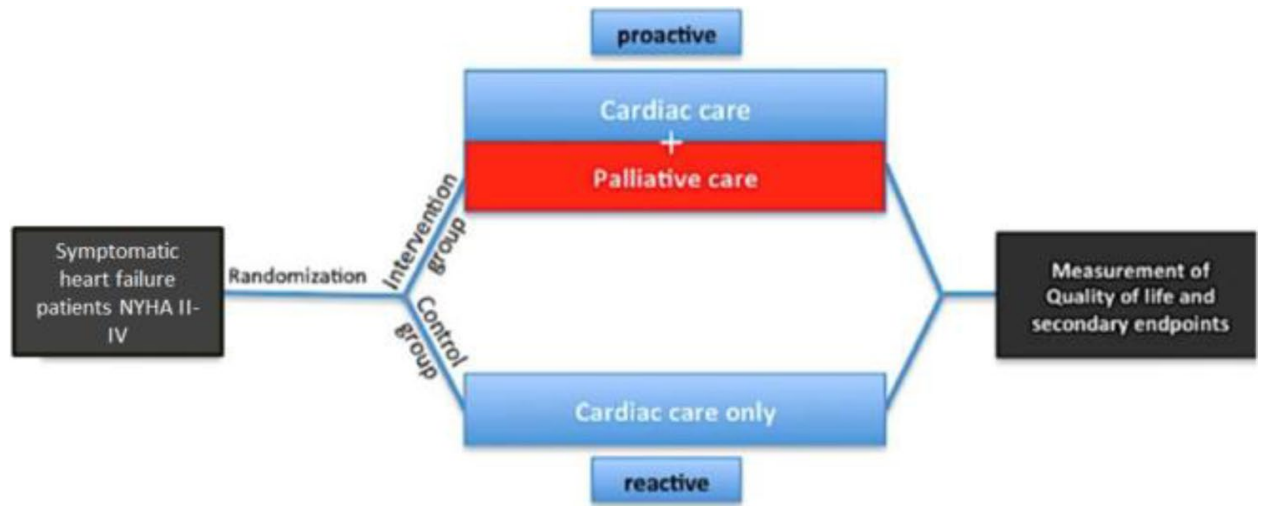

Fig. 1 Intervention scheme of EPCHF. Experimental intervention: additional palliative care to standard cardiac care. The intervention includes monthly consultations by a palliative care team over 12 months (palliative care assessment of physical, psychological, social and spiritual needs, establishing goals of care, assisting with

one of the two groups in a 1:1 ratio to usual contemporary $\mathrm{HF}$ care or usual care combined with the EPCHF intervention (Fig. 1). Subjects are assigned treatment using a complete randomization scheme. Patients who are assigned to early PC will meet with a member of the PC team, which consisted of board-certified PC physicians and advanced-practice nurses within the time of hospitalization after enrollment and at least monthly thereafter in the outpatient setting during the 12 months of follow-up (Figs. 2 and 3). The palliative care intervention focuses on symptom relief, attention to spiritual concerns, and advance care planning. The trial is non-blinded, since it is not possible to execute a double-blinded trial of the EPCHF intervention given that the intervention involves a multidisciplinary team. The duration of the intervention is 12 months, but patients in both groups are followed until death or the end of the study ( 3.5 years). The primary endpoint refers to health-related QoL after 12-month follow-up (Fig. 3).

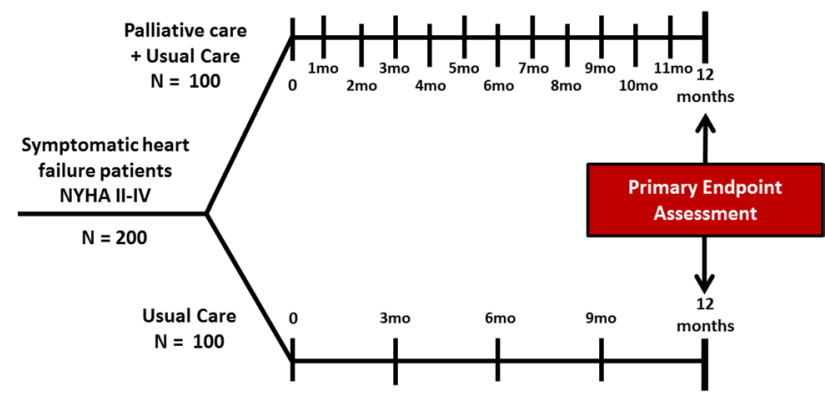

Fig. 2 Trial flow of EPCHF. The individual treatment duration is 12 months. Trial duration: recruiting period: 26 months. Planned start date (FPFV): 01.04.2018. Planned end date (LPLV): 31.07.2021. Time for preparation of the trial (months): 3 months. First patient in to last patient out (months): 38 months. Time for data clearance and analysis (months): 3 months. Duration of the entire trial (months): 42 months decision making) in the outpatient or inpatient setting. Control intervention: standard cardiac care with palliative care on demand only. Duration of intervention per patient: 12 months. Time of data collection: at baseline and at 4 follow-up visits every 3 months within 12 months

\section{Study population}

The study will enroll patients with symptomatic HF defined by dyspnea NYHA $\geq 2$. Inclusion and exclusion criteria are presented in Tables 1 and 2. Mortality risk due to HF will be calculated by the Seattle Heart Failure Score [18].

\section{Usual care}

Patients are managed by a cardiologist-directed team with HF expertise. HF patient care is focused on symptom relief and use of evidence-based therapies [19, 20]. Additional goals of care include treatment of co-morbidities and patient education designed to assist with self-management techniques. For instance, we routinely target sleep disordered breathing and mood disorders, and also encourage exercise training in our HF patients as recommended by guidelines [20]. For ethical reasons, inpatient palliative care consultation at the discretion of the attending cardiologist is not denied to Usual Care patients. HF patients generally receive outpatient follow-up with an HF cardiologist focused on guideline-based medication titration, assessment of adherence to medical and dietary regimens, and serial monitoring of end-organ function. The outpatient palliative care departments of all participating centers are based not readily available to Usual Care patients. As the value of outpatient PC in the HF population is undefined, we believe it is ethically acceptable for outpatients in this arm to receive only stateof-the-art usual cardiovascular care.

\section{Palliative care intervention}

An interdisciplinary, guideline-driven, multi-component PC intervention has been designed and administered with 


\begin{tabular}{|c|c|c|c|c|c|c|c|c|c|c|c|c|c|c|}
\hline & $\begin{array}{c}\text { Visit } \\
1 \\
\text { day }-1 \\
\text { Baseline }\end{array}$ & $\begin{array}{c}\text { Visit } \\
2 \\
\text { dayo }\end{array}$ & $\begin{array}{c}\text { Visit } \\
3 \\
1 \mathrm{mo}\end{array}$ & $\begin{array}{c}\text { Visit } \\
4 \\
2 \mathrm{mo}\end{array}$ & $\begin{array}{c}\text { Visit } \\
5 \\
3 \mathrm{mo}\end{array}$ & $\begin{array}{c}\text { Visit } \\
6 \\
4 \mathrm{mo}\end{array}$ & $\begin{array}{c}\text { Visit } \\
7 \\
5 \mathrm{mo}\end{array}$ & $\begin{array}{c}\text { Visit } \\
8 \\
6 \mathrm{mo}\end{array}$ & $\begin{array}{c}\text { Visit } \\
9 \\
7 \mathrm{mo}\end{array}$ & $\begin{array}{c}\text { Visit } \\
10 \\
8 \mathrm{mo}\end{array}$ & $\begin{array}{c}\text { Visit } \\
11 \\
9 \mathrm{mo}\end{array}$ & $\begin{array}{c}\text { Visit } \\
12 \\
10 \mathrm{mo}\end{array}$ & $\begin{array}{c}\text { Visit } \\
13 \\
11 \mathrm{mo}\end{array}$ & $\begin{array}{c}\text { Visit } \\
14 \\
12 \mathrm{mo}\end{array}$ \\
\hline In-/Exclusion Criteria & $\sqrt{ }$ & & & & & & & & & & & & & \\
\hline Informed Consent & $\checkmark$ & & & & & & & & & & & & & \\
\hline Randomization & & $\sqrt{ }$ & & & & & & & & & & & & \\
\hline Medical History & $\sqrt{ }$ & & & & & & & & & & & & & \\
\hline Vital Signs & $\sqrt{ }$ & $\sqrt{ }$ & & & $\sqrt{ }$ & & & $\sqrt{ }$ & & & $\sqrt{ }$ & & & $\sqrt{ }$ \\
\hline $\begin{array}{l}\text { Physical Examination, ECG, } \\
\text { Echocardiography }{ }^{1}\end{array}$ & $\sqrt{ }$ & $\sqrt{ }$ & & & $\sqrt{ }$ & & & $\sqrt{ }$ & & & $\sqrt{ }$ & & & $\sqrt{ }$ \\
\hline Laboratory tests & $\sqrt{ }^{2}$ & & & & $\checkmark$ & & & $\sqrt{ }$ & & & $\sqrt{ }$ & & & $\sqrt{ }$ \\
\hline Additional Laboratory & $\sqrt{ }$ & & & & $\sqrt{ }$ & & & $\sqrt{ }$ & & & $\sqrt{ }$ & & & $\sqrt{ }$ \\
\hline $\begin{array}{l}\text { completion of medical } \\
\text { history(AEs and SAEs) }\end{array}$ & & $\sqrt{ }$ & & & $\checkmark$ & & & $\checkmark$ & & & $\checkmark$ & & & $\sqrt{ }$ \\
\hline $\begin{array}{l}\text { Seattle HF Model } \\
\text { calculator }\end{array}$ & $\sqrt{ }$ & $\sqrt{ }$ & & & $\checkmark$ & & & $\sqrt{ }$ & & & $\sqrt{ }$ & & & $\sqrt{ }$ \\
\hline HF visits & $\checkmark$ & $\sqrt{ }$ & & & $\sqrt{ }$ & & & $\sqrt{ }$ & & & $\sqrt{ }$ & & & $\sqrt{ }$ \\
\hline $\begin{array}{l}\text { Patient interview } \\
\text { (intervention group) }\end{array}$ & & $\checkmark$ & $\sqrt{ }$ & $\sqrt{ }$ & $\checkmark$ & $\sqrt{ }$ & $\sqrt{ }$ & $\sqrt{ }$ & $\checkmark$ & $\sqrt{ }$ & $\sqrt{ }$ & $\sqrt{ }$ & $\sqrt{ }$ & $\sqrt{ }$ \\
\hline $\begin{array}{l}\text { Palliative care clinical } \\
\text { assessment (interv. group) }\end{array}$ & & $\sqrt{ }$ & $\sqrt{ }$ & $\sqrt{ }$ & $\checkmark$ & $\sqrt{ }$ & $\sqrt{ }$ & $\sqrt{ }$ & $\sqrt{ }$ & $\sqrt{ }$ & $\sqrt{ }$ & $\sqrt{ }$ & $\sqrt{ }$ & $\checkmark$ \\
\hline $\begin{array}{l}\text { Patient preference } \\
\text { assessment (interv. group) }\end{array}$ & & $\checkmark$ & $\sqrt{ }$ & $\sqrt{ }$ & $\sqrt{ }$ & $\sqrt{ }$ & $\sqrt{ }$ & $\sqrt{ }$ & $\sqrt{ }$ & $\sqrt{ }$ & $\sqrt{ }$ & $\sqrt{ }$ & $\sqrt{ }$ & $\sqrt{ }$ \\
\hline FACIT-PAL related QoL (14) & & $\sqrt{ }$ & & & $\sqrt{ }$ & & & $\sqrt{ }$ & & & $\checkmark$ & & & $\sqrt{ }$ \\
\hline KCCQ, related QoL (23) & & $\sqrt{ }$ & & & $\sqrt{ }$ & & & $\sqrt{ }$ & & & $\sqrt{ }$ & & & $\sqrt{ }$ \\
\hline $\begin{array}{l}\text { HADS-D }(14) \\
\text { Depression/Anxiety score }\end{array}$ & & $\checkmark$ & & & $\sqrt{ }$ & & & $\sqrt{ }$ & & & $\sqrt{ }$ & & & $\checkmark$ \\
\hline $\begin{array}{l}\text { MIDOS (8) Symptom } \\
\text { burden score }\end{array}$ & & $\checkmark$ & & & & & & & & & & & & $\sqrt{ }$ \\
\hline $\begin{array}{l}\text { FACIT-Sp (12) Spiritual well } \\
\text { being scale }\end{array}$ & & $\checkmark$ & & & & & & & & & & & & $\checkmark$ \\
\hline $\begin{array}{l}\text { Hospitalization \& death } \\
\text { assessment }\end{array}$ & & & & & & & & & & & & & & $\sqrt{ }$ \\
\hline $\begin{array}{l}\text { Medical resource \& cost } \\
\text { assessment }\end{array}$ & & & & & & & & & & & & & & $\sqrt{ }$ \\
\hline
\end{tabular}

Fig. 3 Schedule of activities. Red font: best supportive cardiac care (BSCC). Blue font: additional early integration of palliative care (EIPC). Black font: conducted investigations. ${ }^{1} \mathrm{LVEF}, \mathrm{LV}$ diastolic

contemporary HF management. The goal is a structured and reproducible approach to assessing and managing the multiple domains of QoL for patients with advanced HF including physical symptoms, psychosocial concerns, spiritual concerns, and advance care planning. At the core of the palliative intervention team is a PC trained NP who coordinates this aspect of the patient's care, a palliative care boardcertified physician, and a trained counselor. Since the same cardiology team cares for patients in both the intervention and control group, the palliative care intervention is performed in collaboration with the cardiology team, but does not involve specific cardiology-based palliative interventions. This approach was designed in an attempt to minimize the extension of PC-specific interventions into the usual care study group to maintain integrity of the study design. Specific attention will be paid to assessing physical and psychosocial symptoms, establishing goals of care, assisting with dysfunction, hemodynamic, ${ }^{2}$ incl. negative blood pregnancy test for women of childbearing potential

decision making regarding treatment, and coordinating care on the basis of the individual needs of the patient [21].

\section{Physical symptoms}

At study enrollment and pre-specified time points, the NP performs standardized assessments to determine the presence of commonly experienced symptoms, including dyspnea, fatigue, pain, anxiety, depression, nausea, anorexia, constipation, and insomnia. Symptoms are managed by the EPCHF palliative team using treatment algorithms to ensure standardization. In addition to periodic assessment and protocolized management of symptoms, patients in both arms are provided with an EPCHF heart failure action plan handout with instruction on regular control of signs that $\mathrm{HF}$ is getting worse and what to do if these changes occur. The handout includes information on self-management of symptoms associated with congestion and on medications 
Table 1 Inclusion criteria

\begin{tabular}{ll}
\hline Inclusion criteria & Subjects will only be included in the study if they meet all of the following criteria \\
\hline Age $\geq 18$ years \\
NYHA class II-IV symptoms (suspected cardiac dyspnea) \\
Systolic HF with a LVEF $\leq 35 \%$ or HF with preserved ejection fraction (NYHA class II-IV, elevated \\
natriuretic peptides (NP), left ventricular hypertrophy (LVH) or left atrial enlargement (LAE) or \\
diastolic dysfunction \\
BNP $\geq 100 \mathrm{pg} / \mathrm{mL}$ or NT proBNP $\geq 400 \mathrm{pg} / \mathrm{mL}$ \\
Subjects with the ability to follow study instructions and likely to attend and complete all required visits \\
Written informed consent
\end{tabular}

Table 2 Exclusion criteria

\begin{tabular}{l}
\hline Exclusion criteria \\
\hline Inability to read, understand and respond to question in German \\
Previous consultations of palliative care services \\
Patients in intensive care unit, on a ventilator, pre- or post-heart transplant \\
Non-cardiac terminal illness \\
Women who are pregnant or planning to become pregnant, nursing women \\
Simultaneously participation in another study or participation in any study \\
involving administration of an investigational medicinal product within 30 \\
days prior to study beginning \\
Subjects with a physical or psychiatric condition which at the investigator's \\
discretion may put the subject at risk, may confound the trial results, or may \\
interfere with the subject's participation in the study \\
Known or persistent abuser of medication, drugs or alcohol \\
\hline
\end{tabular}

typically used for HF on "as needed" basis for symptom relief at home, such as loop diuretics, and sublingual nitroglycerin $[6,22]$. Patients of the interventional arm receive a handout including potential medications that may be prescribed for managing anxiety, nausea, vomiting and constipation. The medications themselves are prescribed at the discretion of the EPCHF clinical provider.

\section{Psychosocial symptoms}

Patients are screened for symptoms of anxiety and depression using the hospital anxiety and depression scale (HADS) [23]. The HADS has been widely used in clinical trials as a psychological screening test for the states of anxiety and depression [24]. Patients screened positive for either anxiety or depression receive more thorough assessment and management as specified by a treatment algorithm used to determine the need for a mental health referral and the possible use of antidepressants, anxiolytics, stress management techniques, and psychotherapy.

\section{Spiritual concerns}

The EPCHF NP completes a spiritual assessment at the time of patient enrollment or at the first outpatient visit after hospitalization using the FICA Spiritual History Tool [25]. FICA is an acronym which serves as a guide and includes: F-Faith and Beliefs; I-Importance in life and health; C-Community-Religious and Spiritual Community; A-How the patient would like spirituality addressed in medical care. The NP shares information gathered in the spiritual assessment with the palliative team and addresses specific spiritual concerns as appropriate. If needed, the NP arranges subsequent visits addressing concerns raised during the initial assessment.

\section{End-of-life preparation}

To address end-of-life preparation, we use the Outlook Intervention which includes three 1 -h sessions, spaced 1 week apart [26]. Session 1 focuses on life review, accomplishments, proudest moments, and cherished times. Session 2 focuses on issues of forgiveness, things the patient would have done differently, and things left unsaid or undone. Session 3 focuses on lessons learned, heritage, and legacy. To ensure replicability, the intervention follows questions outlined in the Outlook Intervention training manual. The Outlook Intervention is administered by the trained counselor. 


\section{Goals of care}

To ensure that the palliative care intervention is specified and replicable, we followed a strict fundamental protocol. Every patient randomized to palliative care was contacted every 28 days with 5-day range of tolerance. The palliative care round was structured by the themes of the modified HOPE questionnaire [27]. Patients in need for help, e.g., with applications have additional appointments by phone or in attendance. In case of symptom management e. g. pain, we contact the cardiologists or GP by phone and gave a recommendation or we involve the pain-OPD to provide prescriptions. Further palliative medical support is highly individual and depends on wishes/topics set by the patient during conversation. The intervention includes communication designed to elicit patients' goals of care which means to determine what is most important to individuals and use those goals to frame discussions of prognosis and of lifeprolonging therapies. These conversations are facilitated by the NP and revisited periodically to document changes in preferences. To ensure standardization of PC treatment, the NP was trained in a 2-day intensive workshop based on the palliative care student's curriculum, originally designed to provide medical students with communication skills to facilitate discussions with seriously ill patients [28]. To assess this aspect of the PC intervention, we document code status, completion of advance directives, and preferences for lifeprolonging therapies.

After the 12-month intervention period is completed, the NP continues to contact the patients in the intervention arm every 3 months to provide ongoing support and clinical care. In addition, the HF team is available for phone consultation to patients randomized to the intervention arm throughout the duration of the trial.

\section{Measurements}

The primary end point is evaluated by health-related qualityof-life questionnaires after 12 months of treatment (Fig. 3). First, the functional assessment of chronic illness therapy palliative care (FACIT-Pal) score evaluating QoL living with a chronic disease and second the Kansas City Cardiomyopathy Questionnaire (KCCQ) measuring QoL living with heart failure will be determined. The FACIT-Pal is a 14-item measure of self-reported QoL that assesses several domains including physical wellbeing, social/family well-being, emotional well-being, and functional well-being [29]. KCCQ is a 23-item, disease-specific questionnaire scored from 0 to 100 with higher scores representing better health status [30]. A 5-point change in the KCCQ overall summary score has been demonstrated to be the minimally detectable clinical difference [26, 28-34].
Secondary end points are changes in anxiety/depression (HADS), symptom burden score (MIDOS), spiritual wellbeing Functional Assessment of Chronic Illness Therapy Spiritual Well-Being Scale (FACIT-Sp), medical resource and cost assessment, and a composite of death, HF hospitalization, and quality of life.

\section{Measures of health care use}

The evaluation of medical resources and costs is carried out using administrative data from the computer system (KAS Orbis) of the electronic medical record of the University Hospital Bonn to analyze costs and resource usage data. The amount paid for care (e. g. through compulsory or private health insurance) should be used as a primary cost estimate. In addition, sensitivity analyzes are performed using the estimated costs of care as a measure of resource use. Differences in the use of resources in the study arms are examined in order not only to determine the extent of the cost differences, but also to identify the sources that make the difference. At all visits, patients are asked if they have been treated outside the study hospital. If so, they are asked to estimate the number of visits and/or days in the hospital. The cost of such care is estimated on the basis of the medical expenditure survey and included in the total cost of care from the time of randomization until the completion of the study.

\section{Data collection}

Participants complete baseline questionnaires before randomization. A complete schedule of assessments is given in Fig. 3. Follow-up evaluations of QoL and mood are expected to occur at 3 months, 6 months, 9 months and 12 months. As necessary, we will mail questionnaires to subjects and/ or complete questionnaires over the telephone to help ensure the collection of primary endpoint data. When responses on questionnaires were incomplete, research staff documented the reasons for which the participant did not give a full response.

\section{Statistical analysis}

Primary endpoints: the two primary endpoints will be tested in a two-step procedure. In the first step the hypothesis of equal scores for the FACIT-Pal in the two treatment groups will be tested with a two-side $t$ test at a level of $5 \%$. In case of the rejection of the null-hypothesis in the first step, the second hypothesis of equal KCCQ-scores in the two treatment groups will be tested again with a two-sided $t$ test at the level of $5 \%$. This test procedure controls a family wise error rate of $5 \%$.

A comparably high number of drop-outs ( 20\%) especially due deaths are to be expected. Since this kind of 
censoring is definitely correlated to the QoL-measurement, the results for the QoL-score might be biased. An optimal choice of a procedure for the replacement of missing values would require a detailed knowledge about this correlation, which is not available. Missing values will not be replaced for the primary analysis of the two primary outcome variables. A sensitivity analysis will be performed replacing missing values by the last observation carried forward method.

For both variables the course of QoL scores over the observational time will analyzed descriptively by fitting mixed linear models to the data. 95\%-confidence interval will be estimated for the score values separated by treatment groups and for measurement times. A second identical analysis for the primary endpoint will be performed based on the per-protocol population (PP), which is defined as the patients treated and observed according to protocol. This analysis will only be performed if ITT and PP-population differ.

Secondary endpoints: the secondary endpoints will be analyzed descriptively in the intention-to-treat collective.

Psychosocial symptoms: patients are screened for symptoms of anxiety and depression using the Hospital Anxiety and Depression Scale (HADS). The HADS has been widely used in studies as a psychologic screening test for the states of anxiety and depression. Patients who screen positive for either anxiety or depression receive a more thorough assessment and management as specified by a treatment algorithm used to determine the need for a mental health referral and the possible use of antidepressants, anxiolytics, stress management techniques, and psychotherapy.

Spiritual concerns: the EPCHF-HF NP completes a spiritual assessment at the time of patient enrolment or at the first outpatient visit after hospitalization using the FACITSp. The NP shares information gathered in the spiritual assessment with the EPCHF team and addresses specific spiritual concerns as appropriate. If needed, the NP arranges for subsequent visits to address concerns raised during the initial assessment.

Moreover, qualitative interview data will supplement the results of questionnaires and gain information about the impact of gender issues and social inequality (e.g., patients with migrant background within the study). In addition, public health researcher with special expertise in the area of health inequality research will analyze the issue between over-indebtedness and HF. Because of the expected relevance of variables pertaining to socioeconomic status and migration background for HF-related outcomes including psychosocial aspects/issues, a careful evaluation of the associations between primary and secondary endpoints and these variables will be investigated.

The secondary endpoints HADS-D, MIDO, FACITSp will be analyzed for differences between the treatment groups by fitting mixed linear models to the data. 95\%-confidence interval will be estimated for the score values separated by treatment groups and for measurement times.

The survival times will be compared between the treatment groups by a log-rank test. Cox-proportional hazard models will be used to further explore the impact of the treatment on the survival of the patients.

The frequency of adverse events will be counted in absolute and relative frequencies, separated by treatment groups and depending on severity and relationship to the therapy.

Further details of the analyses for primary and secondary outcomes will be specified in a statistical analysis plan.

\section{Trial organization}

A Data and Safety Monitoring Board (DSMB) has been appointed to review the conduct and results of this trial at 2 enrollment landmarks (after 33\% and 66\% of the patients have been enrolled). The DSMB is charged with reviewing safety composite data in both arms of the trial. The DSMB will be empowered to stop the study for evidence of harm, but not for evidence of lack of efficacy. The DSMB is also asked to offer perspective on any therapeutic or diagnostic testing advances that may occur during the trial course that may influence the outcome. If protocol modifications are warranted, close consultation among the DSMB and the study leadership will be required.

\section{Summary}

HF creates significant physical, psychosocial and spiritual burden for patients and their families. Palliative care may represent an important component of the holistic management of patients with not only advanced HF. Further evidence from randomized trials of a palliative care intervention in $\mathrm{HF}$ is required to identify which patients may benefit from specific interventions. EPCHF aims to provide empiric data to support the efficacy and cost-effectiveness of palliative care improving the health-related QoL in HF patients after hospitalization. Such evidence could provide impetus to overcome current challenges related to limited access to, and adoption of, palliative care services for HF patients.

Acknowledgements Funded by the Bundesministerium für Bildung und Forschung (BMBF): 01GY17,

https://www.gesundheitsforschung-bmbf.de/de/epchf-studie-fruhepalliativmedizinische-intervention-bei-patientinnen-und-patienten-mit7630.php. Registration No.: DRKS Register 00013922, Studiencode: MED2-201604_EPCHF, Sponsor: BMBF FKZ: 01GY1704, Homepage: EPCHF.de 
Funding Open Access funding enabled and organized by Projekt DEAL.

\section{Declarations}

Conflict of interest No disclosures for the authors.

Open Access This article is licensed under a Creative Commons Attribution 4.0 International License, which permits use, sharing, adaptation, distribution and reproduction in any medium or format, as long as you give appropriate credit to the original author(s) and the source, provide a link to the Creative Commons licence, and indicate if changes were made. The images or other third party material in this article are included in the article's Creative Commons licence, unless indicated otherwise in a credit line to the material. If material is not included in the article's Creative Commons licence and your intended use is not permitted by statutory regulation or exceeds the permitted use, you will need to obtain permission directly from the copyright holder. To view a copy of this licence, visit http://creativecommons.org/licenses/by/4.0/.

\section{References}

1. Go AS, Mozaffarian D, Roger VL et al (2013) Executive summary: heart disease and stroke statistics-2013 update: a report from the American Heart Association. Circulation 127(1):143-152

2. Selman L, Beynon T, Higginson IJ et al (2007) Psychological, social and spiritual distress at the end of life in heart failure patients. Curr Opin Support Palliat Care 1(4):260-266

3. Bekelman DB, Havranek EP, Becker DM et al (2007) Symptoms, depression, and quality of life in patients with heart failure. J Card Fail 13(8):643-648

4. Slaughter MS, Meyer AL, Birks EJ (2011) Destination therapy with left ventricular assist devices: patient selection and outcomes. Curr Opin Cardiol 26(3):232-236

5. Pantilat SZ, Steimle AE (2004) Palliative care for patients with heart failure. JAMA 291(20):2476-2482

6. Goodlin SJ (2009) Palliative care in congestive heart failure. J Am Coll Cardiol 54(5):386-396

7. O'Connor CM, Hasselblad V, Mehta RH et al (2010) Triage after hospitalization with advanced heart failure: the ESCAPE (evaluation study of congestive heart failure and pulmonary artery catheterization effectiveness) risk model and discharge score. J Am Coll Cardiol 55(9):872-878

8. O'Connor CM, Abraham WT, Albert NM et al (2008) Predictors of mortality after discharge in patients hospitalized with heart failure: an analysis from the organized program to initiate lifesaving treatment in hospitalized patients with heart failure (OPTIMIZEHF). Am Heart J 156(4):662-673

9. Goodlin SJ, Hauptman PJ, Arnold R et al (2004) Consensusstatement: palliative and supportive care in advanced heart failure. $\mathrm{J}$ Card Fail 10(3):200-209

10. Yancy CW, Jessup M, Bozkurt B et al (2013) 2013 ACCF/AHA Guideline for the Management of Heart Failure: a report of the American College of Cardiology Foundation/American Heart Association Task Force on Practice Guidelines. J Am Coll Cardiol 62(16):e147-239

11. Evangelista LS, Lombardo D, Malik S et al (2012) Examining the effects of an outpatient palliative care consultation on symptom burden, depression, and quality of life in patients with symptomatic heart failure. J Card Fail 18(12):894-899

12. Evangelista LS, Liao S, Motie M et al (2014) On-going palliative care enhances perceived control and patient activation and reduces symptom distress in patients with symptomatic heart failure: a pilot study. Eur J Cardiovasc Nurs 13(2):116-123

13. Morrison R, Penrod JD, Cassel J et al (2008) Cost savings associated with us hospital palliative care consultation programs. Arch Intern Med 168(16):1783-1790

14. Temel JS, Greer JA, Muzikansky A et al (2010) Early palliative care for patients with metastatic non-small-cell lung cancer. N Engl J Med 363(8):733-742

15. Dalal S, Bruera E (2017) End-of-life care matters: palliative cancer care results in better care and lower costs. Oncologist 22:361-368

16. Kavalieratos D, Corbelli J, Zhang D, Dionne-Odom JN, Ernecoff NC, Hanmer J, Hoydich ZP, Ikejiani DZ, Klein-Fedyshin M, Zimmermann C, Morton SC, Arnold RM, Heller L, Schenker Y (2016) Association between palliative care and patient and caregiver outcomes: a systematic review and meta-analysis. JAMA 316(20):2104-2114

17. Quinn K, Shurrab M, Gitau K et al (2020) With health care use, quality of life, and symptom burden among adults with chronic non-cancer illness: a systematic review and meta-analysis. JAMA 324(14):1439-1450

18. Hunt SA (2005) ACC/AHA 2005 guideline update for the diagnosis and management of chronic heart failure in the adult: a report of the American College of Cardiology/American Heart Association Task Force on Practice Guidelines (Writing Committee to Update the 2001 Guidelines for the Evaluation and Management of Heart Failure). J Am Coll Cardiol 46(6):e1-82

19. Johnson MJ, McDonagh TA, Harkness A et al (2002) Morphine for the relief of breathlessness in patients with chronic heart failure-a pilot study. Eur J Heart Fail 4(6):753-756

20. Zigmond AS, Snaith RP (1983) The hospital anxiety and depression scale. Acta Psychiatr Scand 67(6):361-370

21. Yu DS, Thompson DR, Yu CM et al (2009) Assessing HRQL among Chinese patients with coronary heart disease: angina, myocardial infarction and heart failure. Int J Cardiol 131(3):384-394

22. Borneman T, Ferrell B, Puchalski CM (2010) Evaluation of the FICA tool for spiritual assessment. J Pain Symptom Manage 40(2):163-173

23. Steinhauser KE, Alexander SC, Byock IR et al (2008) Do preparation and life completion discussions improve functioning and quality of life in seriously ill patients? Pilot randomized control trial. J Palliat Med 11(9):1234-1240

24. Fryer-Edwards K, Arnold RM, Baile W et al (2006) Reflective teaching practices: an approach to teaching communication skills in a small-group setting. Acad Med 81(7):638-644

25. Back AL, Arnold RM, Baile WF et al (2007) Efficacy of communication skills training for giving bad news and discussing transitions to palliative care. Arch Intern Med 167(5):453-460

26. Green CP, Porter CB, Bresnahan DR et al (2000) Development and evaluation of the Kansas City cardiomyopathy questionnaire: a new health status measure for heart failure. J Am Coll Cardiol 35(5): $1245-1255$

27. Snyder CR, Harris C, Anderson JR, Holleran SA, Irving LM, Sigmon ST et al (1991) The will and the ways: development and validation of an individual-differences measure of hope. J Pers Soc Psychol 60:570-585

28. Flynn KE, Lin L, Ellis SJ et al (2009) Outcomes, health policy, and managed care: relationships between patient-reported outcome measures and clinical measures in outpatients with heart failure. Am Heart J 158(4 Suppl):S64-71

29. Lyons KD, Bakitas M, Hegel MT et al (2009) Reliability and validity of the Functional Assessment of Chronic Illness Therapy-Palliative care (FACIT-Pal) scale. J Pain Symptom Manage 37(1):23-32 
30. Bekelman DB, Parry C, Curlin FA et al (2010) A comparison of two spirituality instruments and their relationship with depression and quality of life in chronic heart failure. J Pain Symptom Manage 39(3):515-526

31. Peterman AH, Fitchett G, Brady MJ et al (2002) Measuring spiritual well- being in people with cancer: the functional assessment of chronic illness therapy-Spiritual Well-being Scale (FACIT-Sp). Ann Behav Med 24(1):49-58

32. Teno JM, Clarridge B, Casey V et al (2001) Validation of toolkit after-death bereaved family member interview. J Pain Symptom Manage 22(3):752-758
33. Abernethy AP, Kassner CT, Whitten E et al (2011) Death service ratio: a measure of hospice utilization and cost impact. J Pain Symptom Manage 41(6):e5-6

34. Taylor AL, Ziesche S, Yancy C et al (2004) Combination of isosorbide dinitrate and hydralazine in blacks with heart failure. N Engl J Med 351(20):2049-2057 